Pacific

Journal of

Mathematics

LAZARD'S THEOREM FOR DIFFERENTIAL ALGEBRAIC GROUPS AND PROALGEBRAIC GROUPS

Marcin Chalupnik and Piotr Kowalski

Volume $202 \quad$ No. 2

February 2002 


\title{
LAZARD'S THEOREM FOR DIFFERENTIAL ALGEBRAIC GROUPS AND PROALGEBRAIC GROUPS
}

\author{
Marcin Chalupnik and Piotr Kowalski
}

We prove that a differential group whose underlying variety is an affine space is unipotent. The problem is reduced to an infinite-dimensional version of Lazard's Theorem.

\section{Introduction.}

It is well-known that a connected unipotent algebraic group is isomorphic as a variety to an affine space. Lazard ([La]) proved the converse: An algebraic group isomorphic as a variety to an affine space is unipotent. So, the algebraic structure of a unipotent algebraic group is determined by its geometry. Buium and Cassidy $([\mathbf{B C}])$ asked if the same holds for differential algebraic groups, i.e., groups defined by differential polynomials in some differential field. It was proved for groups of small dimension $(=1,2)$ by Cassidy $([\mathbf{C a}])$, and for arbitrary groups over a differentially closed field of characteristic 0 by Kowalski and Pillay $[\mathbf{K P}]$. The principal result of the present paper is the proof of this theorem in the full generality:

Theorem 1. Suppose $(K, D)$ is a differential field. Let $G$ be a differential algebraic group over $K$, with underlying differential variety differentially isomorphic to $A^{n}$. Then $G$ is unipotent (i.e., $G$ may be embedded into a unipotent algebraic group).

The strategy of the proof of this theorem is borrowed from $[\mathbf{K P}]$. We deduce Theorem 1 from a purely algebro-geometric, and interesting for its own right result, which was proved in $[\mathbf{K P}]$ for $K$ an algebraically closed field of characteristic 0 :

Theorem 2. Let $G$ be a group scheme over a field $K$ whose underlying scheme is isomorphic to $A^{\infty}$ (the projective limit of affine spaces). Then $G$ is isomorphic in the category of group schemes over $K$ to an inverse limit of unipotent algebraic groups.

The paper is organized as follows. In Section 2 we reduce Theorem 1 to Theorem 2 (replacing model theory of $[\mathbf{K P}]$ by a simple argument using Hopf algebras). Theorem 2 is proved in Section 4. The proof utilizes in an essential way étale cohomology of schemes and we collect necessary facts concerning it in Section 3. The reader may consult $[\mathbf{B C}]$ for background in 
differential algebraic geometry, while our main reference on étale cohomology is $[\mathbf{M i}]$ (for basics see also very readable [Ta]). The first author would like to thank Andrzej Weber for several enlightening comments concerning étale cohomology. The second author would like to thank Anand Pillay for suggesting this problem, and Ludomir Newelski for careful reading this paper and many helpful remarks.

\section{The reduction.}

Let us fix $(K, D)$ a differential field. $K\{X\}$ denotes the ring of differential polynomials (in a set of variables $X$ ). As a ring $K\{X\}=K\left[X, D X, D^{2} X, \ldots\right]$, where $D^{i} X, i \geq 0$, are tuples of new variables, and derivation on $K\{X\}$ extends $D^{i} X \mapsto D^{i+1} X\left(D^{0} X:=X\right)$. A differential polynomial is an element of $K\{X\}$, and we naturally regard differential polynomials as functions from $K^{n}$ into $K$. We recall some notions from differential algebraic geometry.

\section{Definition 2.1.}

i) A differential algebraic variety is a zero set of a finite number of differential polynomials.

ii) A morphism of differential algebraic varieties is a restriction of a differential polynomial function.

iii) A differential algebraic group is a group object in the category of differential algebraic varieties.

Remark. Usually (e.g., [Bu], [BC]) a morphism of differential algebraic varieties is defined as a function locally given by differential rational functions rather than a globally defined polynomial. However on an affine space these two notions coincide, so we may adopt the more convenient definition.

Lemma 2.2. If $f: G \longrightarrow H$ is a morphism between affine reduced group schemes, then there exist inverse systems $\left(G_{i}\right),\left(H_{i}\right)$ of algebraic groups and a morphism $F$ between them, such that $G=\lim \left(G_{i}\right), H=\lim \left(H_{i}\right)$ and $f=\lim (F)$.

Proof. The proof becomes straightforward when we turn to the category of Hopf algebras, which is dual to the category of affine group schemes. The morphism $f$ corresponds to a morphism $\phi: B \longrightarrow A$, where $B$ is the Hopf algebra corresponding to $H$ and $A$ corresponds to $G$. Since any Hopf algebra is a direct limit of a system of its finitely generated Hopf subalgebras [Wa, p. 24], there exist systems $\left(A_{i}\right)$, and $\left(B_{i}\right)$ of finitely generated reduced Hopf algebras, such that $A=\operatorname{colim}\left(A_{i}\right)$, and $B=\operatorname{colim}\left(B_{i}\right)$. Note that finitely generated reduced Hopf algebras correspond to algebraic groups. Denote by $\phi_{i}^{*}$ the map $\phi$ restricted to the $B_{i}$. Then, since $B_{i}$ is finitely generated, there exists $n_{i}$ such that $\phi_{i}^{*}$ can be factorized through the map $\phi_{i}: B_{i} \longrightarrow A_{n_{i}}$. So $\phi=\lim \left(\phi_{i}\right)$, where $\left(\phi_{i}\right)_{i \in N}$ is a map between direct systems $\left(B_{i}\right)_{i \in N}$ and 
$\left(A_{n_{i}}\right)_{i \in N}$. Going back to the category of affine group schemes we obtain the result.

Remark. A part of Lemma 2.2 (the existence of $\left(G_{i}\right)$ ) was proved in $[\mathbf{K P}]$ by a model-theoretical argument (valid in any stable theory after a suitable reformulation) for algebraic groups over an algebraically closed field.

\section{Corollary 2.3. Theorem 2 implies Theorem 1.}

Proof. Let us consider the ring $K\{X\}$ of differential polynomials in $n$ variables. It is also a ring of differential regular functions (i.e., morphisms into $A^{1}$ ) on $A^{n}$, so as in algebraic geometry, differential group structure on $A^{n}$ gives us the Hopf algebra structure on $K\{X\}$. We denote this Hopf algebra by $A$ (it is also a differential algebra). It corresponds to an infinitedimensional group scheme $G^{*}$. The group $G^{*}(K)$ may be thought of as the set $K^{\infty}$ with the group operation given by the sequence $\left(\mu, D(\mu), D^{2}(\mu), \ldots\right)$, where $D$ is a derivation in $K\{X\}$, and $K\{X\}$ acts on $K^{\infty}$ as the ring of polynomials of infinitely many variables. Then the map $\phi: a \mapsto\left(a, D a, D^{2} a, \ldots\right)$ is a homomorphism between $G$ and $G^{*}(K)$. From Lemma 2.2, we have $G^{*}=\lim \left(G_{i}\right)$, where $G_{i}$ 's are algebraic groups. Denote by $H_{i}$, the Hopf algebra of $G_{i}$. The Hopf algebra $A$ is a direct limit of the system $\left(H_{i}\right)$ and it is finitely generated as a differential algebra, so there exists $n$ such that $H_{n}$ differentially generates $A$. Then the composition morphism $G \longrightarrow G_{n}$ is an embedding, since it induces epimorphism of algebras of differential regular functions. Using Theorem 2 and Lemma 2.2 we see that $G_{n}$ is unipotent.

\section{Remark.}

i) The construction of $G^{*}$ is the same as in $[\mathbf{K P}]$. However here we do not need our field to be differentially closed to check that we obtain a group.

ii) If $G$ is an algebraic group, then $G^{*}$ coincides with the Buium's infinite prolongation, which is an inverse limit of so-called twisted jet spaces $[\mathbf{B u}]$. If $D$ vanishes on the field of definition, then the infinite prolongation of an algebraic group is an inverse limit of the usual jet spaces.

\section{Some étale cohomology of group schemes.}

The aim of this section is to prove some facts about étale cohomology of group schemes which will be needed in the proof of Theorem 2. Throughout this section $\mathbf{k}$ is an algebraically closed field and all schemes are of finite type over $\mathbf{k}$. We are interested in étale cohomology groups with coefficients in the constant sheaf $\mathbf{Z} / l$ where $l$ is a prime distinct from the characteristic. We say that a scheme $X$ is $\mathbf{Z} / l$-acyclic if it has the cohomology of a point i.e., $H_{e t}^{i}(X, \mathbf{Z} / l)=0$ for $i>0$, and $H_{e t}^{0}(X, \mathbf{Z} / l)=\mathbf{Z} / l$; a morphism of schemes 
is called $\mathbf{Z} / l$-acyclic if it induces an isomorphism on $H_{e t}^{*}(-, \mathbf{Z} / l)$. The main computational tool in étale cohomology theory is the Leray spectral sequence associated to a morphism of schemes $f: X \longrightarrow Y$ :

$$
E_{2}^{i j}=H_{e t}^{i}\left(X, \mathbf{R}^{j} f_{*}(\mathbf{Z} / l)\right) \Rightarrow H_{e t}^{i+j}(X, \mathbf{Z} / l) .
$$

The typical application of the Leray spectral sequence is the following criterion of the acyclicity of a morphism:

Lemma 3.1. Let $f: X \longrightarrow Y$ be either proper or smooth. Suppose all fibres of $f$ are $\mathbf{Z} / l$-acyclic. Then $f$ is $\mathbf{Z} / l$-acyclic.

Proof. By the Proper (or Smooth) Base Change Theorem ([Mi], p. 224, p. 230) we may identify stalks of $\mathbf{R}^{j} f_{*}(\mathbf{Z} / l)$ with $j$-th cohomology of fibres. Thus all rows except 0 -th in the Leray spectral sequence disappear. Now it suffices to observe that since $f$ has connected fibre, then $\mathbf{R}^{0} f_{*}(\mathbf{Z} / l)=$ $\mathbf{Z} / l$.

If $f$ is locally trivial (i.e., locally a projection from a product) and $Y$ is smooth, then the Leray spectral sequence takes the form known from algebraic topology. We recall that a scheme is simply connected if it has trivial algebraic fundamental group (i.e., the group classifying étale coverings of a scheme).

Lemma 3.2. Suppose $f: X \longrightarrow Y$ is locally trivial in the étale topology, and $Y$ is smooth, connected and simply connected. Denote by $F$ the fibre of $f$. Then the Leray spectral sequence associated with $f$ has the following form:

$$
E_{2}^{i j}=H_{e t}^{i}\left(X, H_{e t}^{j}(F, \mathbf{Z} / l)\right) \Rightarrow H_{e t}^{i+j}(X, \mathbf{Z} / l),
$$

where $H_{e t}^{j}(F, \mathbf{Z} / l)$ is a constant sheaf.

Proof. From the Smooth Base Change Theorem ([Mi], p. 230) we derive that if $f$ is a projection, then $\mathbf{R}^{j} f_{*}(\mathbf{Z} / l)$ may be identified with a constant sheaf having a stalk $H_{e t}^{j}(F, \mathbf{Z} / l)$, where $F$ is a fibre of $f$. So for $f$ locally trivial, the sheaf $\mathbf{R}^{j} f_{*}(\mathbf{Z} / l)$ is locally trivial with a stalk $H_{e t}^{j}(F, \mathbf{Z} / l)$. However, analogously to the classical context, a locally constant sheaf with finite stalks (we point out that $H_{e t}^{j}(F, \mathbf{Z} / l)$ for smooth $F$ is always finite ([Mi], p. 244)) on a connected scheme $Y$ is determined by an action of algebraic fundamental group of $Y$ on a stalk ([Mi], p. 156). Thus for a simply connected scheme $Y$ a locally constant sheaf with finite stalks must be constant and the Leray spectral sequence has the required form. 
Another useful spectral sequence is the Hochschild-Serre spectral sequence corresponding to a Galois covering $p: X \longrightarrow Y$ with the structural group $G$ ([Mi], p. 105):

$$
E_{2}^{i j}=H^{i}\left(G, H_{e t}^{j}(X, \mathbf{Z} / l)\right) \Rightarrow H_{e t}^{i+j}(Y, \mathbf{Z} / l),
$$

here $H^{i}(G,-)$ denotes cohomology of the discrete group $G$. Now we turn to the facts we need for the proof of Theorem 2 .

Proposition 3.3. Let $f: G \longrightarrow H$ be an isogeny of connected affine algebraic groups. Then for almost all primes $l, f$ is $\mathbf{Z} / l$-acyclic.

Proof. Let $K$ be the kernel of $f$ regarded as a finite affine group scheme. Then it may be obtained as an extension: $1 \longrightarrow K^{0} \longrightarrow K \longrightarrow \Pi_{0}(K) \longrightarrow$ 1 , where $K^{0}$ is connected and $\Pi_{0}(K)$ is étale ([Wa $]$, p. 51). Hence we may factorize $f$ as

$$
G \stackrel{f_{c}}{\longrightarrow} G / K^{0} \stackrel{f_{e t}}{\longrightarrow}\left(G / K^{0}\right) / \Pi_{0}(K)=H,
$$

where $f_{c}$ has connected fibres and $f_{e t}$ is a Galois covering.

Since $K^{0}$ as a scheme is just a point (in general with multiplicity), fibres of $f_{c}$ are $\mathbf{Z} / l$-acyclic. Moreover $f_{c}$ being finite must be proper. Thus it satisfies the assumptions of Lemma 3.1, hence is $\mathbf{Z} / l$-acyclic for any $l$.

Let us turn to $f_{e t}$ and take $l$ prime to $\left|\Pi_{0}(K)\right|$. Then the Hochschild-Serre spectral sequence corresponding to $f_{e t}$ degenerates and we get $H_{e t}^{*}(H, \mathbf{Z} / l)=$ $\left(H_{e t}^{*}\left(\left(G / K^{0}\right), \mathbf{Z} / l\right)\right)^{\Pi_{0}(K)}$, which gives us a monomorphism $H_{e t}^{*}(H, \mathbf{Z} / l) \longrightarrow$ $H_{e t}^{*}\left(\left(G / K^{0}\right), \mathbf{Z} / l\right)$. This monomorphism is compatible with $f_{e t}^{*}$ by the very construction of the spectral sequence. Now it remains to show that $\Pi_{0}(K)$ acts trivially on $H_{e t}^{*}\left(\left(G / K^{0}\right), \mathbf{Z} / l\right)$. But the action of $\Pi_{0}(K)$ on $G / K^{0}$ extends to the action of the whole group $G / K^{0}$, which is connected. Thus the triviality of the action at the level of cohomology will follow if we show that any embedding $\phi_{g}: G / K^{0} \longrightarrow G / K^{0} \times G / K^{0}$ defined by $\phi_{g}(h)=(h, g)$ induces the same morphism on cohomology as $\phi_{e}$. The last fact follows immediately form the Kunneth formula for $G / K^{0} \times G / K^{0}$ ([SGA], p. 236), and the trivial observation that two constant morphisms into a connected scheme induce the same on étale cohomology.

Proposition 3.4. Let $G$ be a simple group. Then for almost all primes $l$, $G$ is not $\mathbf{Z} /$ l-acyclic.

Proof. First observe that $G$ has finite algebraic fundamental group. Indeed, it suffices to show that there is only a finite number of étale coverings of $G$. But this follows from the fact that any étale covering is étale (hence central) isogeny, while we have only a finite number of possibilities for centers of groups having root systems isomorphic to the root system of $G([\mathbf{H u}], \mathrm{p}$. 215 ), and all these centers are finite. Thus $G$ has the universal covering being an étale isogeny. Therefore, according to Proposition 3.3, we may assume 
that $G$ is simply connected. Let $B \subset G$ be a Borel subgroup. Then the sequence $B \longrightarrow G \longrightarrow G / B$ is locally trivial in the étale topology [Se], and $G / B$ is simply connected (this follows from $\Pi_{1}^{\text {alg }}(G)=0$ and connectivity of $B)$. Then, by Lemma 3.2 we have:

$$
E_{2}^{i j}=H_{e t}^{i}\left(G / B, H_{e t}^{j}(B, \mathbf{Z} / l)\right) \Rightarrow H_{e t}^{i+j}(G, \mathbf{Z} / l) .
$$

Now take $i_{0}=\max \left\{i: H_{e t}^{i}(G / B, \mathbf{Z} / l) \neq 0\right\}$. Since G/B is projective, it has the fundamental class ([Mi], pp. 247-252), so $i_{0}=2 \cdot \operatorname{dim}(G / B)>0$. Similarly we take $j_{0}=\max \left\{j: H_{e t}^{j}(B, \mathbf{Z} / l) \neq 0\right\}$. Then we have $0 \neq E_{2}^{i_{0} j_{0}}=$ $E_{\infty}^{i_{0} j_{0}}$, hence $H_{e t}^{i_{0}+j_{0}}(G, \mathbf{Z} / l) \neq 0$, so $G$ is not $\mathbf{Z} / l$-acyclic.

\section{The proof of Theorem 2 .}

The main ingredient in the proof is the following:

Proposition 4.1. Let $f: G \longrightarrow Q$ be an epimorphism of connected affine algebraic groups defined over an algebraically closed field $\mathbf{k}$, and assume that $f$ factorizes through an affine space. Then $Q$ is solvable.

Proof. Suppose $Q$ is not solvable. Then we have an epimorphism $p: Q \longrightarrow S$ with simple $S$, hence we may assume that $Q$ is already simple. According to the acyclicity of $\mathbf{A}^{n}$ ([Mi], p. 295) and Proposition 3.4 in order to obtain contradiction it suffices to show that $f$ induces a monomorphism on étale cohomology with coefficients in $\mathbf{Z} / l$ for almost all $l$ 's.

Let $R_{u}$ be the unipotent radical of $G$. Then since $R_{u} \subset \operatorname{ker}(f)$, we may factorize $f$ as $G \stackrel{g}{\longrightarrow} G / R_{u} \stackrel{h}{\longrightarrow} Q$. Let us first investigate $g$. Observe that $g$ is smooth and has acyclic fibres, since $R_{u}$ as a variety is isomorphic to an affine space. Thus according to Lemma 3.1 it induces an isomorphism on étale cohomology for any $l$ prime to the characteristic. So, we may focus on $h$. Since $G / R_{u}$ is reductive we have an isogeny $i: T \times S_{1} \times \ldots \times S_{n} \longrightarrow G / R_{u}$, where $T$ is a torus and each $S_{i}$ is simple. We shall see that $(h \circ i)^{*}$ is a monomorphism on étale cohomology for some $l$. Indeed, since $Q$ is simple, there exists such $j$ that $\left.(h \circ i)\right|_{S_{j}}: S_{j} \longrightarrow Q$ is also an isogeny. Hence $\left(\left.(h \circ i)\right|_{S_{j}}\right)^{*}$ is an isomorphism for almost all l's, by Proposition 3.3. Thus $(h \circ i)^{*}\left(\right.$ a fortiori $\left.h^{*}\right)$ is a monomorphism for these $l$ 's.

In fact we need the following, quite straightforward, generalization of the above proposition:

Corollary 4.2. Let $K$ be any field, and $f: G \longrightarrow Q$ be an epimorphism of connected affine algebraic groups defined over $K$. Suppose that $f$ factors through an affine space. Then $Q$ is unipotent. 
Proof. Let $G_{L}=G \otimes L, Q_{L}=Q \otimes L, f_{L}=f \otimes L$, where $L$ is the algebraic closure of $K$. Then the epimorphism $f_{L}: G_{L} \longrightarrow Q_{L}$ factors through an affine space, so by Proposition 4.1, $Q_{L}$ is solvable. Moreover, any connected solvable group is a semidirect product of its unipotent radical $R$ and some algebraic torus $\left([\mathbf{H u}]\right.$, p. 123). But since $Q_{L}$ is an image of an affine space, it cannot be mapped onto a torus. Thus $Q_{L}$ is unipotent. It is also easy to see that $Q_{L}$ is unipotent iff $Q$ is (see e.g., [Wa], p. 64). This completes the proof.

Now we are in a position to prove our main theorem. We recall that by Corollary 2.3 it suffices to prove Theorem 2 .

Proof of Theorem 2. Let $G$ be a group scheme whose underlying scheme is $A^{\infty}$. By Lemma 2.2, $G$ is isomorphic to an inverse limit of a system $\left(G_{i}\right)$ of algebraic groups. It is enough to prove that $G_{i}$ 's are unipotent. Fix a positive integer $n$. Then, like in the proof of Lemma 2.2 , the isomorphism between $G$ and $\lim \left(G_{i}\right)$ yields morphisms $f: G_{N} \longrightarrow A^{l}$, and $g: A^{l} \longrightarrow G_{n}$, for some $l$ and $N>n$. The composition $g \circ f$ is an epimorphism, since it comes from the identity map on $G$. From Corollary $4.2, G_{n}$ is unipotent.

\section{References}

[Bu] A. Buium, Geometry of differential polynomial functions I: Algebraic groups, Amer. J. Math., 115 (1993), 1385-1444, MR 95c:12011, Zbl 0797.14016.

[BC] A. Buium and Ph.J. Cassidy, Differential algebraic geometry and differential algebraic groups, to appear in collected works of E. Kolchin.

[Ca] Ph.J. Cassidy, Unipotent differential algebraic groups, in 'Contributions to algebra', Academic Press, (1977), 83-115, MR 80m:14033, Zbl 0389.12011.

[SGA] P. Deligne, J.F. Boutot, A. Grothendieck, L. Ilusie and J.L. Verdier, SGA $4 \frac{1}{2}$ Cohomologie Étale, Lectures Notes in Math., 569, Springer, Heidelberg, 1977, MR 57 \#3132, Zbl 0349.14008.

[Hu] J. Humphreys, Linear Algebraic Groups, Springer, New York, 1981, MR 82c:20080, Zbl 0471.20029.

[KP] P. Kowalski and A. Pillay, Proalgebraic and differential algebraic group structures on affine spaces, Amer. J. Math., 122(1) (2000), 213-221, MR 2001d:14049.

[La] M. Lazard, Sur nilpotence de certains groupes algebriques, C.R.A.S. Paris, 241 (1955), 1687-1689, MR 17,710a, Zbl 0065.25401.

[Mi] J. Milne, Étale Cohomology, Princeton University Press, Princeton, 1980, MR 81j:14002, Zbl 0433.14012.

[Se] J.P. Serre, Espaces fibrés algébriques, Séminaire Chevalley, 2 (1958), Exposé 1, Secrétariat Mathématique, Paris, MR 31 \#1277, Zbl 0132.41202.

[Ta] G. Tamme, Introduction to Étale Cohomology, Springer, Berlin, 1994, MR 95k:14033, Zbl 0815.14012. 
[Wa] W. Waterhouse, Introduction to Affine Group Schemes, Springer, New York, 1979, MR 82e:14003, Zbl 0442.14017.

Received April 13, 2000 and revised July 10, 2000. The first author was partially supported by Bialynicki-Birula Subsydium of Foundation of Polish Science and the polish scientific grant (KBN) 2 P03A 002 18. The second author was partially supported by the polish scientific grant (KBN) 2 P03A 02018.

INSTYTUT MATEMATYKI

UNIVERSITY OF WARSAW

UL. BANACHA 2, 02-097 WARSAW

POLAND

E-mail address: mchal@mimuw.edu.pl

INSTYTUT MATEMATYCZNY

UNIVERSITY OF WROC€AW

PL. GRunWALDZKI $2 / 4$

50-384 WrocŁaW

POLAND

E-mail address: pkowa@math.uni.wroc.pl 